$11-2-2017$

\title{
Efficient full wave code for the coupling of large multirow multijunction LH grills
}

Josef Preinhaelter

preinh@ipp.cas.cz

Julien Hillairet

Daniele Milanesio

Riccardo Maggiora

Jakub Urban

See next page for additional authors

Follow this and additional works at: https://scholarworks.wm.edu/aspubs

\section{Recommended Citation}

Preinhaelter, Josef; Hillairet, Julien; Milanesio, Daniele; Maggiora, Riccardo; Urban, Jakub; Vahala, Linda; and Vahala, George, Efficient full wave code for the coupling of large multirow multijunction LH grills (2017). NUCLEAR FUSION, 57(11).

10.1088/1741-4326/aa7f4f

This Article is brought to you for free and open access by the Arts and Sciences at W\&M ScholarWorks. It has been accepted for inclusion in Arts \& Sciences Articles by an authorized administrator of W\&M ScholarWorks. For more information, please contact scholarworks@wm.edu. 
Authors

Josef Preinhaelter, Julien Hillairet, Daniele Milanesio, Riccardo Maggiora, Jakub Urban, Linda Vahala, and George Vahala 
PAPER

\section{Efficient full wave code for the coupling of large multirow multijunction LH grills}

To cite this article: Josef Preinhaelter et al 2017 Nucl. Fusion 57116060

View the article online for updates and enhancements.
Related content

An analysis of lower hybrid grill coupling
$\frac{\text { using an efficient full wave code }}{\text { Josef Preinhaelter, Jakub Urban, Linda }}$
Vahala et al.
ALOHA: an Advanced LOwer Hybrid
$\frac{\text { Antenna coupling code }}{\text { J. Hillairet, D. Voyer, A. Ekedahl et al. }}$
- Experimental characterization and
$\frac{\text { modelling of non-linear coupling of the }}{\text { lower hybrid current drive power on Tore }}$
$\frac{\text { Supra }}{\text { M. Preynas, M. Goniche, J. Hillairet et al. }}$




\title{
Efficient full wave code for the coupling of large multirow multijunction LH grills
}

\author{
Josef Preinhaelter ${ }^{1}$, Julien Hillairet ${ }^{2}$, Daniele Milanesio ${ }^{3}$, \\ Riccardo Maggiora ${ }^{3}$, Jakub Urban ${ }^{1} \oplus$, Linda Vahala ${ }^{4}$ and George Vahala ${ }^{5}$ \\ ${ }^{1}$ Institute of Plasma Physics of the CAS, Za Slovankou 1782/3, 18000 Prague, Czech Republic \\ 2 CEA, IRFM, F-13108 Saint Paul-lez-Durance, France \\ 3 Dipartimento di Elettronica, Politecnico Di Torino, Torino, Italy \\ 4 Old Dominion University, Norfolk, VA 23529, United States of America \\ ${ }^{5}$ College of William \& Mary, Williamsburg, VA 23185, United States of America \\ E-mail: preinh@ipp.cas.cz
}

Received 17 September 2015, revised 19 April 2017

Accepted for publication 12 July 2017

Published 1 September 2017

\begin{abstract}
The full wave code OLGA, for determining the coupling of a single row lower hybrid launcher (waveguide grills) to the plasma, is extended to handle multirow multijunction active passive structures (like the C3 and C4 launchers on TORE SUPRA) by implementing the scattering matrix formalism. The extended code is still computationally fast because of the use of (i) 2D splines of the plasma surface admittance in the accessibility region of the $k$-space, (ii) high order Gaussian quadrature rules for the integration of the coupling elements and (iii) utilizing the symmetries of the coupling elements in the multiperiodic structures. The extended OLGA code is benchmarked against the ALOHA-1D, ALOHA-2D and TOPLHA codes for the coupling of the C3 and C4 TORE SUPRA launchers for several plasma configurations derived from reflectometry and interferometery. Unlike nearly all codes (except the ALOHA-1D code), OLGA does not require large computational resources and can be used for everyday usage in planning experimental runs. In particular, it is shown that the OLGA code correctly handles the coupling of the $\mathrm{C} 3$ and $\mathrm{C} 4$ launchers over a very wide range of plasma densities in front of the grill.
\end{abstract}

Keywords: lower hybrid waves, coupling, large multirow multijunction grills, tokamak, full-wave

(Some figures may appear in colour only in the online journal)

\section{Introduction}

Lower hybrid (LH) waves, whose frequencies lie between the electron and ion cyclotron frequencies, are an excellent candidate for current drive in tokamaks. However, these waves cannot be launched directly into the plasma because the cut-off density is very low and lies at the plasma edge. To avoid this problem, spatially slow down waves are utilized since they can propagate as slow waves in the dense plasma behind the cutoff. Typically, these waves are launched by waveguide grills and there exist fast efficient codes that determine the coupling of these antennas to the plasma. A survey of earlier work on LH coupling is described in our earlier papers [1] and [2]. Here we mention only those codes which scale appropriately when moving from a single-row to large multi-row multi-junction LH grills as C3 [3] and C4 [4] for TORE SUPRA or envisaged launcher for ITER [5]. Codes such as ALOHA-1D, ALOHA-2D [2], TOPLHA [6] and GRILL3D [7] can handle these large multi-row multi-junction grills and they are being run on possible ITER LH launcher scenarios $[8,9]$. The $3 \mathrm{D}$ full wave finite element code LHEAF [10] (see also [11]) solves the coupling of the launcher as well as the interaction of LH waves with the ITER plasma. However all these codes, with the exception of the ALOHA-1D code, require large computational resources, and being relatively slow are rather impractical for everyday usage in planning experimental runs.

Our goal is to fill this gap by updating our fast code OLGA [1] and testing it on the large passive-active multi-junction 
launchers C3 and C4 on TORE SUPRA. We shall outline the new features that have been introduced into the OLGA code in order for it to be able to handle these large antenna structures while remaining a fast and efficient code.

In section 2 we will first examine the effect of several rows of waveguides on the coupling of the LH grill to the plasma. Modern LH launchers consist of multi-junction sections and these can be represented by scattering matrices. These scattering matrices are determined from the 3D finite element solution of wave propagation in the corresponding waveguide junction (for example, on using the commercially available package ANSYS HFSS [12]). These matrices include the curvature of the grill mouth designed to the magnetic surface in front of the grill. From the 1D full wave model of wave propagation in the plasma used in OLGA we can approximate the grill coupling in 3D space (a similar approach is employed in the TOPLHA code). We analyze the role of the accessibility [13] of these launched waves on the power spectrum directionality and the connected current drive efficiency of the grill, using both WKB and full wave calculations. The situation is further complicated by the final depth of integration in the full wave solution of the wave propagation which leads to the reduced accessibility limit.

In section 3, we benchmark our OLGA code against ALOHA-1D, ALOHA-2D and TOPLHA in the coupling of the TORE SUPRA C3 and C4 launchers to the plasma. In the TORE SUPRA shot 43016, we examine 9 experimental density profiles whose density in front of the grill systematically grows from $n_{\text {mouth }}=0.2 n_{\text {crit }}$ to $3 n_{\text {crit }}$ (for LH wave frequency $f=3.7 \mathrm{GHz}$ the critical density for the slow wave cutoff is $n_{\text {crit }}=1.69 \times 10^{17} \mathrm{~m}^{-3}$ ). This density range covers the parameter regime from ineffective to optimal coupling. We discuss simulation results for the OLGA and ALOHA-1D codes for the coupling of the $\mathrm{C} 3$ and $\mathrm{C} 4$ launchers. We also consider a so-called reduced launcher ( 2 central modules from the upper row and 3 passive waveguides) using not only OLGA and ALOHA-1D, but also the ALOHA-2D and TOPLHA codes.

Finally, in appendix, the symmetry properties of the coupling elements for the multi-periodic system are expounded.

\section{Extension of the OLGA code to simulate multi-row multi-junction launchers}

Our earlier OLGA code [1] could only model simple LH wave launchers. Here we will extend the OLGA code to handle multi-row multi-junction launchers while still retaining a highly efficient code capable of quick wallclock turnaround as needed in rapid experimental feedback.

We use the same notation as in [1], with coordinate system: origin at the geometric center of the grill mouth, $z$ parallel to the magnetic field $B, y$ in the poloidal direction ('up'), and $x$ directed radially into the plasma. Thus the radial, poloidal and toroidal components of the wave refractive index emitted by the launcher are $N_{x}, N_{y}, N_{z}$. We shall also orient the long sides of the waveguides to be perpendicular to the magnetic field, and employ Gaussian units with harmonic dependence $\mathrm{e}^{-\mathrm{i} \omega t}$. The plasma is modeled by a simple $1 \mathrm{D}$ algorithm.

\subsection{Multi-row system}

The LH wave launchers are considered to have $N_{\text {rows }}$ identical rows, numbered from the top to the bottom, with $y_{\text {irow }}$ denoting the positions of the upper horizontal walls of the waveguides in the $i$ th row. Since the integrands of the coupling elements between waveguide modes in different rows are proportional to the highly oscillatory factor $\exp \left(\mathrm{i} N_{y} k_{\text {vac }}\left(y_{\text {irow }}-y_{\text {jrow }}\right)\right)$, the coupling between modes in different waveguides is very weak. Thus the 1D spectrum (formed by integrating the spectral power density over $N_{y}$ ) for a one row system is essentially the same as for a multi-row system with the same geometric waveguides.. So in ALOHA-1D, when we study the C3 and C4 launchers, we restrict ourselves to just one row of multijunction modules. In OLGA, the inclusion of the coupling between rows does not lead to a significant degradation of the code's performance. However, it should be mentioned that in a real experiment the poloidal inhomogeneity can have an important effect on the coupling of the multirow system. Also the multirow multijunction modules exhibit very significant coupling between the rows of the modules.

Multirow effect are seen in the 2D spectrum. The 2D spectrum for a 1-row system forms a series of peaks located along the line $N_{y}=0$. For the multi-row system each peak splits in the poloidal direction into several peaks located at $N_{y}=(\Delta \Phi+2 \pi s) /\left(k_{\text {vac }}\left(a+d_{\text {row }}\right)\right), s=0, \pm 1, \pm 2, \ldots$,where $a$ is the height of the waveguides, $d_{\text {row }}$ is the width of the septum between the rows, $k_{\mathrm{vac}}=\omega / c$ is the vacuum wave number, and $\Delta \Phi$ is the phase shift between the waves powering waveguides in different rows.

\subsection{Multi-junction modules and the scattering matrixes}

It is convenient to first consider the scattering matrix formulation on a simple 1-row, 4-waveguide multi-junction module operating at $3.7 \mathrm{GHz}$ and with a built in phase shift of $\pi / 2$. To apply the standard junction theorem [14], we must assume that the mouth of the grill and the mouth of the power waveguide must be well separated from all irregularities inside the junction. Under these conditions there are no nonpropagating modes (which modes could originate in the junction) and one needs only to consider the $\mathrm{TE}_{10}$ modes here. The scattering matrix connects the incoming and outgoing wave amplitudes.

For a simple multi-junction grill, one obtains the following incident wave amplitudes on the plasma

$$
\begin{aligned}
A_{10, p}^{H}= & \frac{\pi}{a k_{\mathrm{vac}}} \sum_{g=2}^{N_{w_{-} \mathrm{MJ}}+1} S_{\left(N_{w_{-} \mathrm{MJ}}-p+2\right), g}^{*} B_{10, N_{w_{-} \mathrm{MJ}}-g+2}^{H} \\
& +S_{\left(N_{w_{-} \mathrm{MJ}}-p+2\right), 1}^{*} \sqrt{\frac{b^{\mathrm{MJ}}}{b}} \mathrm{e}^{\mathrm{i} \phi^{0}} \mathrm{~A}_{10,0}^{H} .
\end{aligned}
$$

Here, $A_{10, p}^{H}, B_{10, p}^{H}, p=1, \ldots, 4$ are the amplitudes of the incident and reflected $\mathrm{TE}_{10}$ modes in the waveguides at the grill mouth, $\mathrm{A}_{10,0}^{H}$ is the amplitude of the incident $\mathrm{TE}_{10}$ mode from power (active) waveguide, $a$ and $b$ are the height and width of the waveguides at the grill mouth while $a^{\mathrm{MJ}}, b^{\mathrm{MJ}}$ are the height and width of the power waveguide. It should 


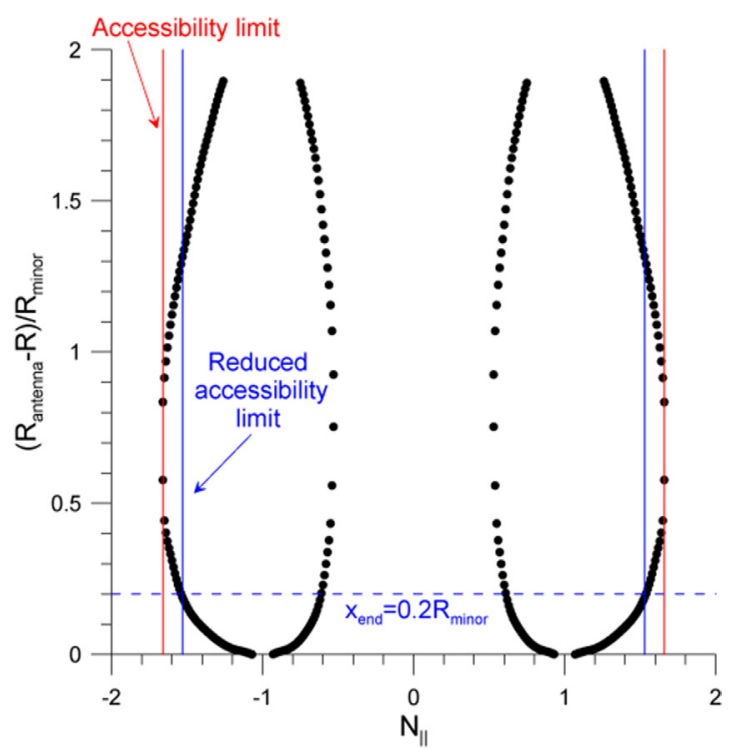

Figure 1. The radial positions of the slow to fast wave branch crossings versus $N_{\|}$. Accessibility limit $N_{\|}=1.66$, while the reduced accessibility limit $N_{\|}=1.53$. C3 launcher, profile \# 9 .

be noted that the orthonormal form of the waveguide modes (see (6) in [2]) used to determine the scattering matrix $\mathbf{S}$ differs from the form of these modes used here and in OLGA (see (A.3) in [1]). This dictates the form of the coefficients in (1). Since we use time dependence of $\mathrm{e}^{-\mathrm{i} \omega t}$ in OLGA, the appropriate scattering matrix in (1) is $\mathbf{S}^{*}$ rather than $\mathbf{S}$. Also, in OLGA we reverse the notation of waveguide positions from that used in the derivation of $\mathbf{S}$.

When $A_{10, p}^{H}$ is inserted into the right hand sides of the original system to determine the coefficients of the transverse electric and magnetic modes in the waveguides $B_{m, n, p}^{H}, B_{m, n, p}^{E}$ ((A.19) and (A.20) in [1]) we obtain a new right hand sides proportional to $A_{10,0}^{H}$ as well as corrections to the coefficients at $B_{10, p}^{H}$.

For the reflected waves in the power waveguides we have:

$$
\begin{aligned}
\frac{\pi}{a^{\mathrm{MJ}} k_{\mathrm{vac}}} \sqrt{\frac{b^{\mathrm{MJ}}}{b}} \mathrm{e}^{\mathrm{i} \varphi^{0} \mathrm{~B}_{10,0}^{H}=} & S_{11}^{*} \sqrt{\frac{b^{\mathrm{MJ}}}{b}} \mathrm{e}^{\mathrm{i} \varphi^{0}} \mathrm{~A}_{10,0}^{H} \\
& +\frac{\pi}{a k_{\mathrm{vac}}} \sum_{g=2}^{N_{w_{-} \mathrm{MJ}}+1} S_{1, g}^{*} B_{10, N_{w_{-}} \mathrm{MJ}-g+2}^{H} .
\end{aligned}
$$

The power reflected by one section of the multi-junction grill is determined by $\mathrm{B}_{10,0}^{H}$.

\subsection{Accessibility limit}

The accessibility limit, $N_{\|}^{\text {access }}$, of LH waves in a plasma is readily determined from the WKB solution for wave propagation in a cold plasma. A radial search of the zeros of the discriminant of the bi-quadratic equation for $N_{x}^{2}$ determines $N_{\|}$and the radial position at which the slow and fast waves are coupled (figure 1). Our integration domain is $-5 \leqslant N_{\text {pol }} \leqslant 5$ and $0 \leqslant N_{\|} \leqslant 30$. For $\left|N_{\text {pol }}\right| \leqslant 5$ and if $N_{\|}<N_{\|}^{\text {access }}$ both the fast and slow waves propagate and are coupled. The accessibility limit is independent of $N_{\text {pol }}$ and is symmetric in the

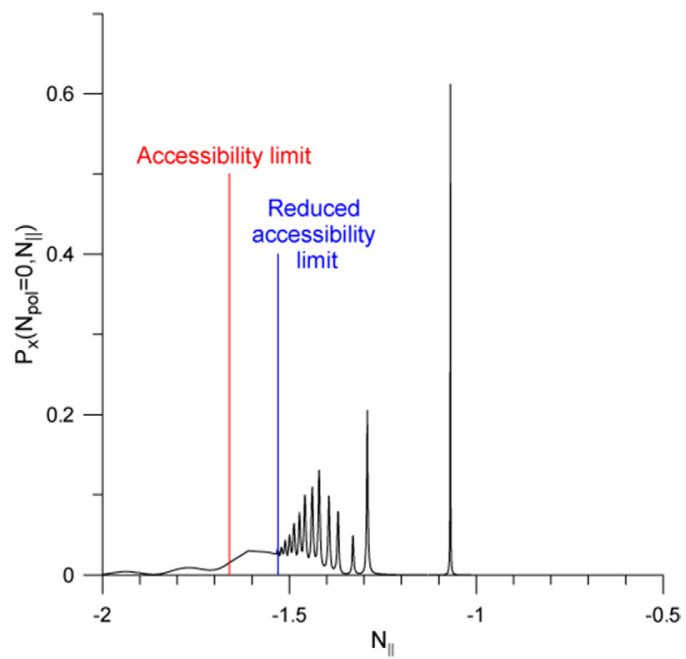

Figure 2. The spectral power density versus $N_{\|}$and the location of the accessibility limits. The sharp peaks correspond to the individual plasma slab eigenmodes.

interchange $N_{\|} \rightleftarrows-N_{\|}$. We assume that the waves generated in the inaccessibility region do not penetrate to the plasma center [15], but are lost with respect to current drive and just collisionally heat the boundary plasma.

When performing a full wave solution we can only integrate the wave equations to some finite depth $x_{\text {end }}$. The reduced accessibility limit, $N_{\|, \text {reduced }}^{\text {access }}$, corresponds to this depth $x_{\text {end }}$ and waves with $N_{\|}>N_{\| \text {,reduced }}^{\text {access }}$ are decoupled. For TORE SUPRA, $x_{\text {end }}=0.2 R_{\text {minor }} \sim 14 \mathrm{~cm}$ looks optimal. We find the numerical integration to be quite fast and the neglected effects of the toroidal and poloidal curvatures to be small with the first eigenmode fully damped. The accessibility limit can be also determined from the full wave solution. One simple method is to determine the eigenmode with the largest $N_{\|}$on the cut $N_{\text {pol }}=0$ of the spectral power density (figure 2). For larger $x_{\text {end }}$ the eigenmodes with high $N_{\|}$are difficult to detect but the slow waves below the accessibility limit are still coupled to the fast waves.

It is desired to have the main coupling results to be independent of the model parameter $x_{\text {end }}$. This holds for the power reflection coefficient. We shall show later that for optimal coupling the important parameters that depend on the accessibility limit (such as directivity and the power lost in the inaccessible region) saturate at $x_{\text {end }}=0.2 R_{\text {minor }}$ and do not change with increasing $\mathrm{x}_{\text {end }}$.

\subsection{Indicators of the coupling efficiency}

In particular, the most important indicator is the power reflection coefficient $C^{\mathrm{PR}}=P_{\text {reflected }}^{\text {tot }} / P_{\text {incident }}^{\text {tot }}$. Because of the accessibility limit we must modify various indicators to describe the coupling efficiency. The power transmission coefficient of the traveling waves (the slow waves reaching the plasma center) is denoted by

$$
C^{\mathrm{PTW}}=\int_{-\infty}^{\infty} \mathrm{d} N_{\text {pol }}\left(\int_{N_{\|}^{\text {access }}}^{\infty}+\int_{-\infty}^{-N_{\|}^{\text {access }}}\right) P\left(N_{\text {pol }}, N_{\|}\right) \mathrm{d} N_{\|} .
$$




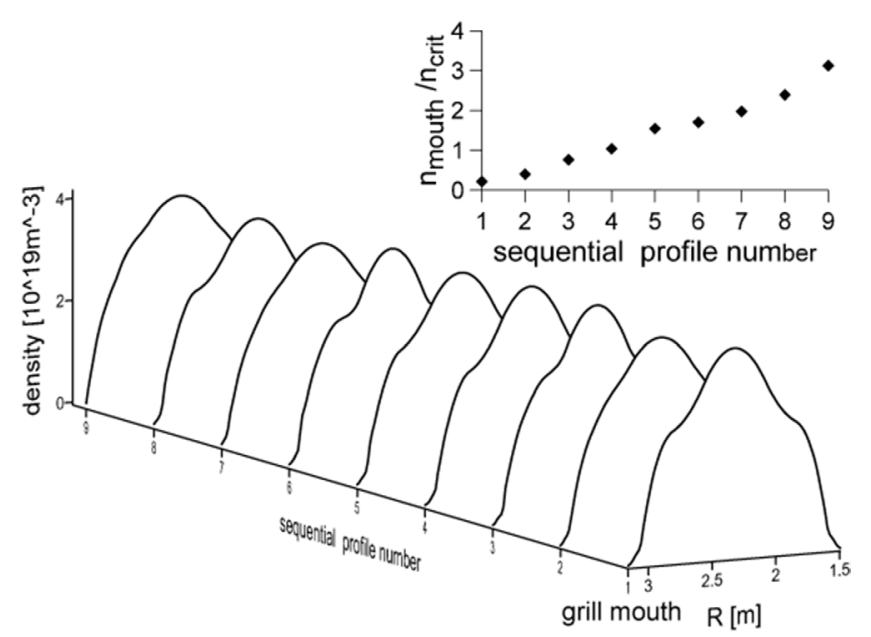

Figure 3. Nine radial density profiles used in the benchmarking OLGA against ALOHA-1D, ALOHA-2D and TOPLHA. The grill mouth is located at $R=3.1583 \mathrm{~m}$. In the inset (upper right corner) are shown the corresponding 9 normalized densities at the grill mouth

Here, and also in figures 2, 6, 8 and $11, P\left(N_{\text {pol }}, N_{\|}\right)$is the time averaged spectral power density normalized to the total time averaged power incident from the generators.

$C^{\mathrm{PCW}}$ is the normalized power lost due to wave coupling (slow $\rightleftarrows$ fast waves):

$$
C^{\mathrm{PCW}}=\int_{-\infty}^{\infty} \mathrm{d} N_{\text {pol }} \int_{-N_{\|}^{\text {access }}}^{N_{\|}^{\text {access }}} P\left(N_{\text {pol }}, N_{\|}\right) \mathrm{d} N_{\|}
$$

This arises from the plasma slab eigenmodes (i.e. the coupled standing slow and fast waves), from the traveling coupled, slow-fast, waves in the inaccessible region with $\left|N_{\|}\right| \leqslant N_{\|}^{\text {access }}$ as well as from the vacuum waves. That part of the power radiated as standing vacuum waves (the fast waves reflected at the left cutoff $\left.\left(n=n_{\text {crit }}\left(1+f_{c e} / f\right)\left(1-N_{\|}^{2}\right)\right)\right)$ is given by

$$
C^{\mathrm{PVW}}=\int_{-1}^{1} \mathrm{~d} N_{\mathrm{pol}} \int_{-\sqrt{1-N_{\mathrm{pol}}^{2}}}^{\sqrt{1-N_{\mathrm{pol}}^{2}}} P\left(N_{\mathrm{pol}}, N_{\|}\right) \mathrm{d} N_{\|} .
$$

Typically, the conservation of power flow in the OLGA code is maintained to 5 digits, if we take into account the losses in the multijunctions of launcher.

The simple directivity of the transmitted traveling waves can be defined:

$$
D^{\mathrm{TW}}=\int_{-\infty}^{\infty} \mathrm{d} N_{\mathrm{pol}} \int_{N_{\|}^{\text {access }}}^{\infty} P\left(N_{\mathrm{pol}}, N_{\|}\right) \mathrm{d} N_{\|} / C^{\mathrm{PTW}}
$$

However, a more important quantity is the $N_{\| \text {-weighted }}$ directivity [16], since this describes better the generation of toroidal current:

$$
\begin{aligned}
D_{c d}= & \int_{-\infty}^{\infty}\left(\int_{N_{\|}^{\text {access }}}^{\infty} P\left(N_{\text {pol }}, N_{\|}\right)\left(N_{\|}^{\text {peak }}\right)^{2} / N_{\|}^{2} \mathrm{~d} N_{\|}\right. \\
& \left.-\int_{-\infty}^{-N_{\|}^{\text {access }}} P\left(N_{\text {pol }}, N_{\|}\right)\left(N_{\|}^{\text {peak }}\right)^{2} / N_{\|}^{2} \mathrm{~d} N_{\|}\right) \mathrm{d} N_{\text {pol }}
\end{aligned}
$$

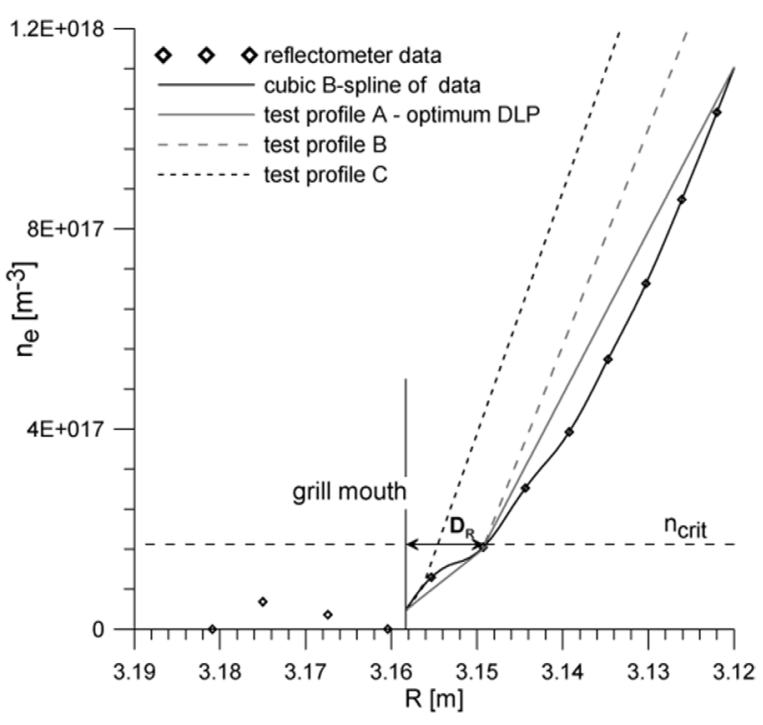

Figure 4. The details of the density profile \#1 at the grill mouth.

Here $N_{\|}^{\text {access }}$ can be either the full accessibility or the reduced accessibility and $N_{\|}^{\text {peak }}$ is the position of the main peak in the transmitted spectrum. $P\left(N_{\mathrm{pol}}, N_{\|}\right)$is the time averaged spectral power density normalized to the total time averaged power incident from the generators.

\section{Benchmarking OLGA with ALOHA and TOPLHA CODES}

We benchmark our OLGA code with ALOHA-1D, ALOHA-2D and TOPLHA on a series of 9 specific plasma density profiles from shot \#43016 on TORE SUPRA (figure 3) for the $\mathrm{C} 3$ and $\mathrm{C} 4$ launchers. The profiles are selected so that the plasma surface density at the grill mouth grows from $0.2 n_{\text {crit }}$ (for the 1 st profile) to $3 n_{\text {crit }}$ (for the 9 th profile). This wide range of plasma densities permits us to compare the coupling of the launchers from bad to optimal conditions. The density profiles from the grill mouth to the plasma center are obtained from a combination of interferometry and reflectometry measurements.

Of the four codes considered in our benchmarking, only the OLGA code can directly utilize the spline interpolation of the experimental density profile. In the other codes (ALOHA-1D, ALOHA-2D, TOPLHA), the density profile is assumed to be linear. Moreover, in ALOHA-2D and TOPLHA one utilizes only a single electron density gradient $\nabla n$ to account for the density following the step density $n_{\text {mouth }}$ at the mouth of the waveguides, while in ALOHA-1D one considers a profile with 2 density gradients $\nabla n_{1}, \nabla n_{2}$ (double linear density profile).

To determine the optimal double linear density profile (DLP) for each experimental profile, we use the OLGA code and compare the power reflection coefficient of the $\mathrm{C} 3$ launcher (see below) determined from the original profile with the power reflection coefficient corresponding to the selected test linear profile. For optimal DLP parameters, there should be an excellent agreement in determining $C^{\mathrm{PR}}$.

We must thus determine the 2 density gradients $\nabla n_{1}, \nabla n_{2}$ as well as the transition point $D_{R}$ for each particular density 
Table 1. Parameters for the optimal double linear profiles (DLP) and power reflection coefficients $\left(C^{\mathrm{PR}}\right)$ for original (splined reflectometer data) profiles (OLGA), for optimal DLP (OLGA) and for optimal DLP (ALOHA-1D). This is for the C3 launcher with the magnetic field on the axis, $B_{0}=3.98 \mathrm{~T}$. $D_{R}$ is the radial distance from the grill mouth at which there is a transition from linear density gradient $\nabla n_{1}$ to the gradient $\nabla n_{2}$ (see figure 4 ).

\begin{tabular}{lllllll}
\hline Profile no. & $n_{\text {mouth }}$ & $\nabla n_{1}$ & $\nabla n_{2}$ & $D_{R}$ & $C_{\mathrm{OLGA}}^{\mathrm{PR}}$ & $C_{\mathrm{OLGA}-\mathrm{DLP}}^{\mathrm{PR}}$ \\
\hline 1 & $3.8 \times 10^{+16}$ & $1.4 \times 10^{+19}$ & $3.3 \times 10^{+19}$ & 0.00916 & 41.4925 & 40.7094 \\
2 & $6.9 \times 10^{+16}$ & $2.2 \times 10^{+19}$ & $4.1 \times 10^{+19}$ & 0.01167 & 27.3118 & 25.5839 \\
3 & $1.3 \times 10^{+17}$ & $1.7 \times 10^{+19}$ & $4.2 \times 10^{+19}$ & 0.02044 & 19.3736 & 14.7944 \\
4 & $1.8 \times 10^{+17}$ & $1.8 \times 10^{+19}$ & $3.9 \times 10^{+19}$ & 0.03031 & 10.3805 & 9.00323 \\
5 & $2.6 \times 10^{+17}$ & $1.4 \times 10^{+19}$ & $3.4 \times 10^{+19}$ & 0.00505 & 5.27917 & 5.06441 \\
6 & $2.9 \times 10^{+17}$ & $2.2 \times 10^{+19}$ & $4.0 \times 10^{+19}$ & 0.01417 & 4.01755 & 3.65227 \\
7 & $3.4 \times 10^{+17}$ & $4.4 \times 10^{+19}$ & $1.7 \times 10^{+20}$ & 0.04214 & 2.94766 & 2.66059 \\
8 & $4.1 \times 10^{+17}$ & $3.1 \times 10^{+19}$ & $5.9 \times 10^{+19}$ & 0.03633 & 2.6947 & 2.63 \\
9 & $5.3 \times 10^{+17}$ & $9.6 \times 10^{+19}$ & $2.1 \times 10^{+20}$ & 0.00535 & 2.27401 & 3.05615 \\
\hline
\end{tabular}

profile. For profile \#1 we consider 3 linear test profiles, shown in figure 4 . The linear profile A connects the density at the grill mouth $n_{\text {mouth }}$ with the intersection of the profile with the critical density $n_{\text {crit }}$, while the second spatial gradient is chosen to fit the first several centimeters of the experimental profile. The first part of linear profile B (the dashed in figure 4) is identical with profile $\mathrm{A}$ and its second linear section connects the position of $n_{\text {crit }}$ with the maximum density at $R_{\text {major }}$. The dotted line in figure 4 is the linear density profile $\mathrm{C}$ where the first gradient $\nabla n_{1}=\mathrm{d} n\left(R_{\text {mouth }}\right) / \mathrm{d} x, D_{R}$ is chosen to be $2 \mathrm{~mm}$, and the second gradient $\nabla n_{2}$ is so chosen that the profile passes through $n\left(R_{\text {major }}\right)$. However, if $n_{\text {mouth }} \geqslant n_{\text {crit }}$, one selects $\nabla n_{1}, \nabla n_{2}$ and $D_{R}$ to optimize the linear profile fit with the experimental profile.

The density profile A used in OLGA produces practically the same $C^{\mathrm{PR}}$ as the original profile and so represents the optimum DLP (see table 1). The density profile B, chosen to reproduce the correct central density peak, gives only slightly worse results. This confirms that the density profiles deeper into the plasma will have only a weak effect on the launcher coupling. The density profile $\mathrm{C}$ based on the local density gradient at the grill mouth gives totally incorrect results (a $C^{\mathrm{PR}}=4.5 \%$ instead $41.5 \%$ for the splined measured profile). This confirms that the main parameters affecting the launcher coupling are $n_{\text {mouth }}$ and the depth of the evanescent region for waves with $\left|N_{\|}\right|>1$. However, the strength of the local density gradient near the grill mouth has a negligible effect on the coupling (apart from giving information on the purely linear profile). For $n_{\text {mouth }}>n_{\text {crit }}$, the coupling is determined principally by $n_{\text {mouth }}$ and in all cases there is an obvious weak dependence of the coupling on the shape of the density profile near the grill mouth (approximately $1-2 \mathrm{~cm}$ from the mouth).

It should be noted that for some profiles it is difficult to determine an optimal DLP. For example, for profile \#3 (table 1) there are large variations in the density gradient near the grill mouth and these are very difficult to model with just a double linear profile.

When we are testing the coupling, we consider ideal launching conditions so that the incident power in all the modules are the same and the phases of the incident waves are at their optimal values. The real experimental effects associated with fluctuations in the power and phase are neglected, and we only consider the effects arising from the actual density and magnetic field profiles on the launcher-plasma coupling. In determining the coupling for the OLGA code, we restrict ourselves to 12 waveguide modes $\left(\mathrm{TE}_{01}, \mathrm{TE}_{02}, \mathrm{TE}_{10}, \mathrm{TE}_{11}, \mathrm{TE}_{12}\right.$, $\mathrm{TE}_{20}, \mathrm{TE}_{21}, \mathrm{TE}_{22}, \mathrm{TM}_{11}, \mathrm{TM}_{12}, \mathrm{TM}_{21}, \mathrm{TM}_{22}$ )—which is more than sufficient. In ALOHA-1D we consider only one row of modules. In ALOHA-2D and TOPLHA we use only $\nabla n_{1}$ from the optimal DLP.

\subsection{C3 launcher}

The C3 launcher on TORE SUPRA consists of 16 multijunction modules arranged in 2 rows. Each module is composed of 3 rows of waveguides (six waveguides in each row with the $90^{\circ}$ built-in phase shift). The modules are separated by 3 passive waveguides arranged in columns. Incident waves (at $f=3.7 \mathrm{GHz}$ ) in the power-supplied waveguides of the individual modules have a phase difference of $270^{\circ}$.

We first computed the power reflection coefficients of the C3 launcher for the 9 measured density profiles in table 1 from the OLGA code and compared them to those computed from the ALOHA-1D code using the optimal DLP, figure 5. We see that both the OLGA and ALOHA-1D codes yield similar results for the coupling of the $\mathrm{C} 3$ launcher, independent of the density at the grill mouth. The major difference occurs for density profile \#3 (see table 1) and this can be attributable to the inadequacy of the double linear profile in representing the actual density profile. One sees not only that the reflection coefficients from the 2 codes are in fairly good agreement, but also the spectral power densities (figure 6). For density profile \#9 this spectral power density has 2 very narrow peaks which are located in the accessibility region. This excellent agreement between OLGA and ALOHA-1D arises because the radiated spectrum is predominantly in the slow waves: fast waves are ignored in ALOHA-1D.

Because of this excellent agreement in the radiated spectrum from the OLGA and ALOHA-1D codes for the profile \#9, one sees excellent agreement in the power reflection coefficients $\left(C_{\mathrm{OLGA}}^{\mathrm{PR}}=2.27 \%, C_{\mathrm{ALOHA}-1 \mathrm{D}}^{\mathrm{PR}}=2.2 \%\right)$ as well as in the power transmitted to the traveling waves $\left(C_{\mathrm{OLGA}}^{\mathrm{PTW}}=92 \%\right.$, $\left.C_{\mathrm{ALOHA}-1 \mathrm{D}}^{\mathrm{PTW}}=91.6 \%\right)$ and the power lost to the inaccessible 


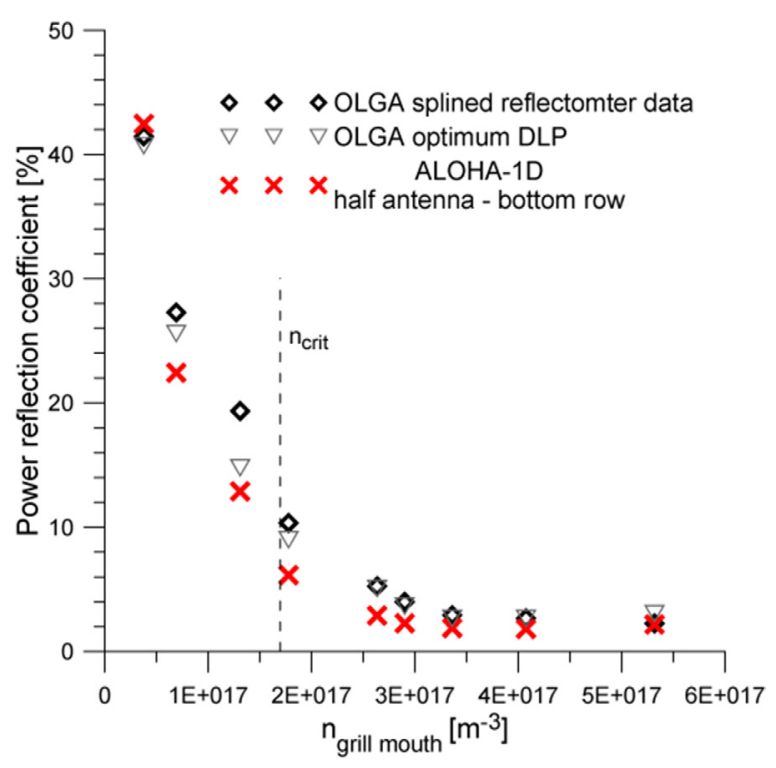

Figure 5. The power reflection coefficient of the $\mathrm{C} 3$ launcher: benchmarking OLGA and ALOHA-1D for the 9 density profiles of figure 3.

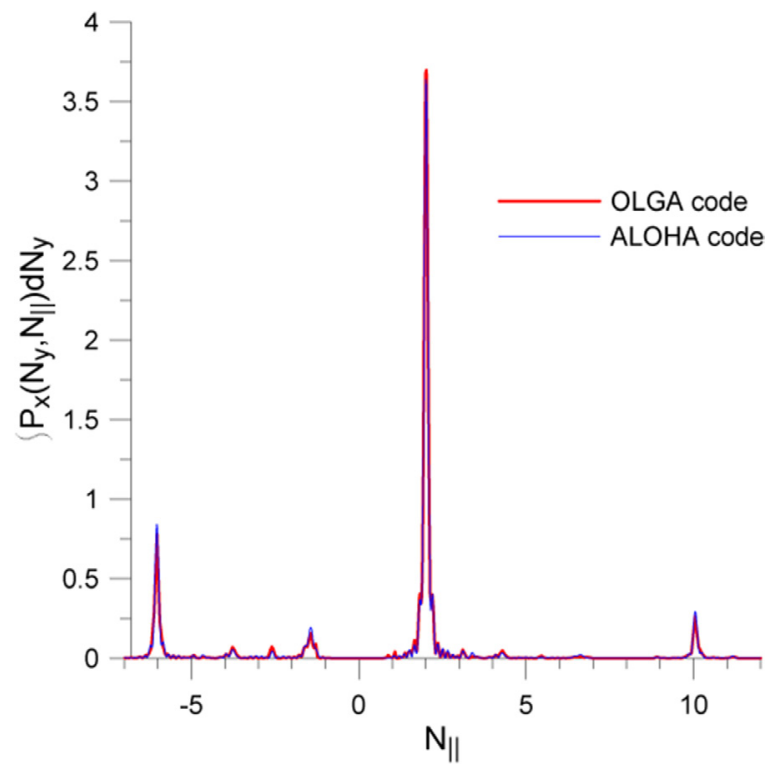

Figure 6. Comparison of the spectral power density integrated over $N_{\text {pol }}$ from OLGA and ALOHA-1D for the C3 launcher, with optimal coupling conditions from density profile $\# 9$ of figure 3

region $\left(C_{\mathrm{OLGA}}^{\mathrm{PCW}}=5.6 \%, C_{\mathrm{ALOHA}-1 \mathrm{D}}^{\mathrm{PCW}}=5.9 \%\right)$. The main peak at $N_{\|}^{\max }=2$ contains a substantial part of the radiated power and both codes predict practically the same directivity of the traveling waves $\left(D_{\mathrm{OLGA}}^{\mathrm{TW}}=78 \%, D_{\mathrm{ALOHA}-1 \mathrm{D}}^{\mathrm{TW}}=77.8 \%\right)$ as well as the same weighted directivity $\left(D_{c d}^{\mathrm{OLGA}}=61 \%\right.$, $\left.D_{c d}^{\mathrm{ALOHA}-1 \mathrm{D}}=58.5 \%\right)$. In the ALOHA results we have used the accessibility limit as derived from the OLGA code $\left(N_{\|}^{\text {access }}=1.66\right)$ and derived the above coefficients using the ALOHA spectral density profile integrated over $N_{\text {pol }}$.

For the validity of the OLGA results themselves, it is important that the reduced accessibility limit $\left(N_{\| \text {,reduced }}^{\text {access }}=1.53\right)$ has little effect on these coefficients. Using this reduced accessibility limit, we find $D_{\text {reduced }}^{\mathrm{TW}}=77.6 \%, D_{\text {cd }}^{\text {reduced }}=60.7 \%$,

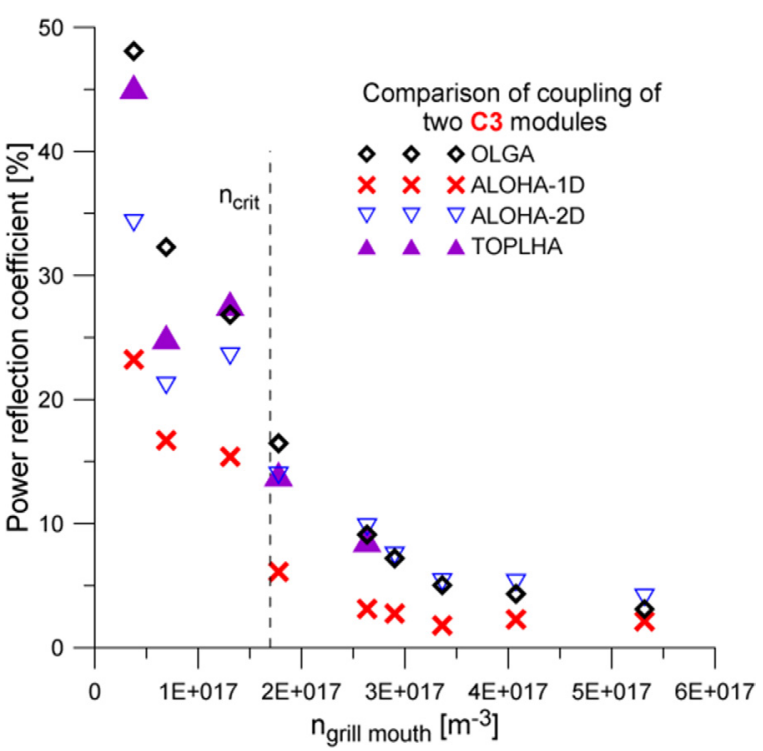

Figure 7. Benchmarking the power reflection coefficient for the 9 density profiles (figure 3 and table 1) from OLGA and ALOHA-1D, ALOHA-2D and TOPLHA for the reduced C3 launcher.

$C_{\text {reduced }}^{\mathrm{PCW}}=4.1 \%$. The results are saturated at $x_{\mathrm{end}}=0.2 R_{\text {minor }}$, with insensitivity to the choice of the model parameter $x_{\text {end }}$.

Both codes give essentially the same coupling coefficients and nearly identical spectral power densities also at the end of the discharge when the plasma column has retreated from the grill mouth and $n_{\text {mouth }}$ is subcritical (profile \#1). The coupling in this case is bad, with high power reflection coefficients $\left(C_{\mathrm{OLGA}}^{\mathrm{PR}}=41 \%, C_{\mathrm{ALOHA}-1 \mathrm{D}}^{\mathrm{PR}}=42.5 \%\right)$. Only a small part of the incident wave penetrates to the plasma center $\left(C^{\mathrm{PTW}}=33 \%\right)$, and even though the simple directivity remain high $\left(D^{\mathrm{TW}}=78 \%\right.$ ) the current drive efficiency is low ( $D_{c d}=19.5 \%$ ). A large part of the power is radiated into the inaccessible region $\left(C^{\mathrm{PCW}}=25.5 \%\right)$ but the $\mathrm{C} 3$ launcher does not radiate vacuum waves $\left(C^{\mathrm{PVW}}=1.5 \%\right)$.

The reduced accessibility limit is rather low in this case $\left(N_{\|, \text {reduced }}^{\text {acess }}=1.39\right.$ in comparison with $\left.N_{\|}^{\text {access }}=1.63\right)$ and the reduced parameters that characterize the coupling are well off the saturation in this case $\left(C_{\text {reduced }}^{\mathrm{PTW}}=41 \%, D_{\text {reduced }}^{\mathrm{TW}}=63 \%\right.$, $\left.C_{\text {reduced }}^{\mathrm{PCW}}=17 \%, D_{c d}^{\text {reduced }}=11 \%\right)$. These results do not improve even when $x_{\text {end }}$ is increased to $x_{\text {end }}=0.5 R_{\text {minor }}$. However, what is important is that the non-reduced coupling parameters $\left(C^{R P}, C^{\mathrm{PTW}}, D^{\mathrm{TW}}, C^{\mathrm{PCW}}\right.$ and $\left.D_{c d}\right)$ are not dependent on $x_{\mathrm{end}}$. In the following, we shall assume that the coupling is completely characterized by these non-reduced parameters.

Finally, we have tested the coupling arising from a reduced C3 launcher. Such a structure will permit a comparison between OLGA (and ALOHA-1D) with those from ALOHA-2D and TOPLHA. We see from figure 7 that ALOHA-1D systematically predicts a lower power reflection coefficient than the other codes. This can be attributed to the limitations of only permitting slow waves in ALOHA-1D as well as the rather broad spectral peaks arising from the reduced short launcher structure. The outgoing slow waves in the inaccessibility region are not coupled to the reflected fast waves leading to an overestimated transmitted power. The power reflection coefficients from OLGA and ALOHA-2D are essentially in 


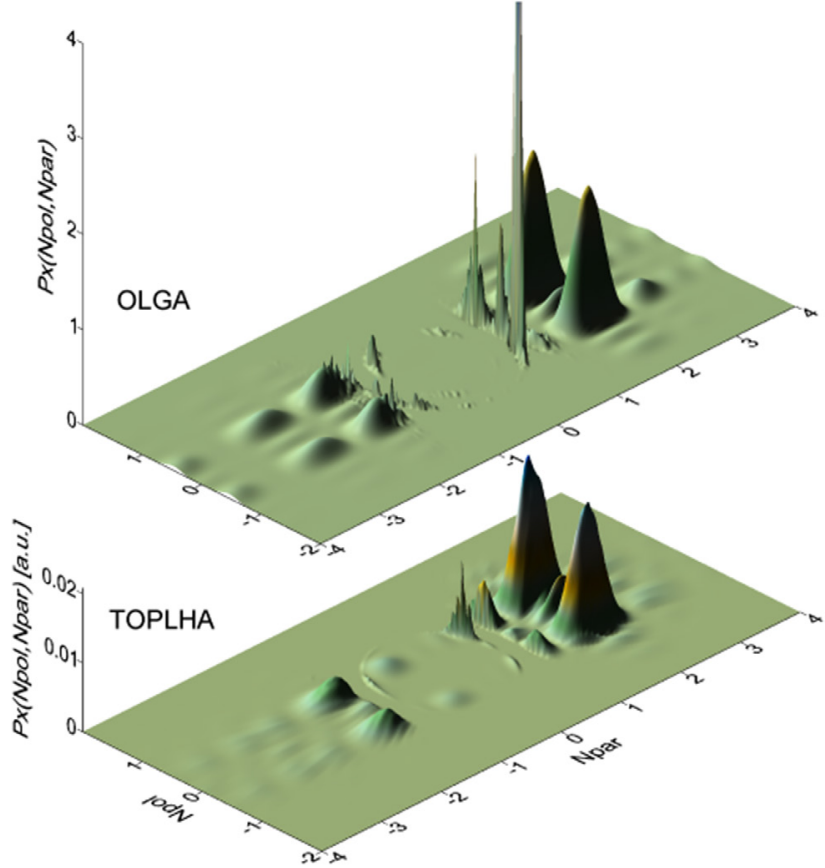

Figure 8. Comparison of 2D spectral power densities of 2 central C3 modules computed by TOPLHA and OLGA for the density profile \#5.

total agreement when $n_{\text {mouth }} \geqslant n_{\text {crit }}$. However, if the density in front of the grill is subcritical and the power in the inaccessible region is increased one finds in ALOHA-2D that the uncoupled slow waves will again transmit too much power into the plasma interior.

To verify OLGA results for coupling in the region, where $n_{\text {mouth }}<n_{\text {crit }}$, we need to benchmark against a code that uses the full wave solution for wave propagation in the plasma and can accept the scattering matrix formalism. The TOPLHA code is well suited for this purpose. As seen in figure 7 , the power reflection coefficient computed by TOPLHA coincides with that of OLGA (the profiles \# 1 , $3-5$ ) or it is the closest to the OLGA result when compared with the other codes (the profile \# 2). In figure 8 we show that not only $C^{\mathrm{PR}}$ but also the $2 \mathrm{D}$ spectral power densities determined by TOPLHA and OLGA are very similar. In the inaccessibility region, TOPLHA code predicts lower level of the plasma slab eigenmodes (the space resonant slow to fast coupled waves). This is understandable because only in the OLGA code is there an iterative search for the eigenmodes. Also the subsidiary peaks at $N_{\|}= \pm 3$ are in TOPLHA figure weaker because it uses only the fundamental waveguide mode.

The peaks of the 2D spectrum of the spectral power density in the region of accessibility are split poloidally into two main peaks. This is a consequence of $\mathrm{TE}_{10}-\mathrm{TE}_{30}$ mode converter used in the $\mathrm{C} 3$ and $\mathrm{C} 4$ launchers as a poloidal divider. In this way three rows of waveguides in the multijunction section are powered with $180^{\circ}$ phase shift. Such an arrangement excites the symmetric spectrum in the poloidal direction, as shown in section 2.1. In the region of inaccessibility we see the first plasma slab eigenmodes encircle the $N_{y}^{2}+N_{z}^{2}=1$ region.

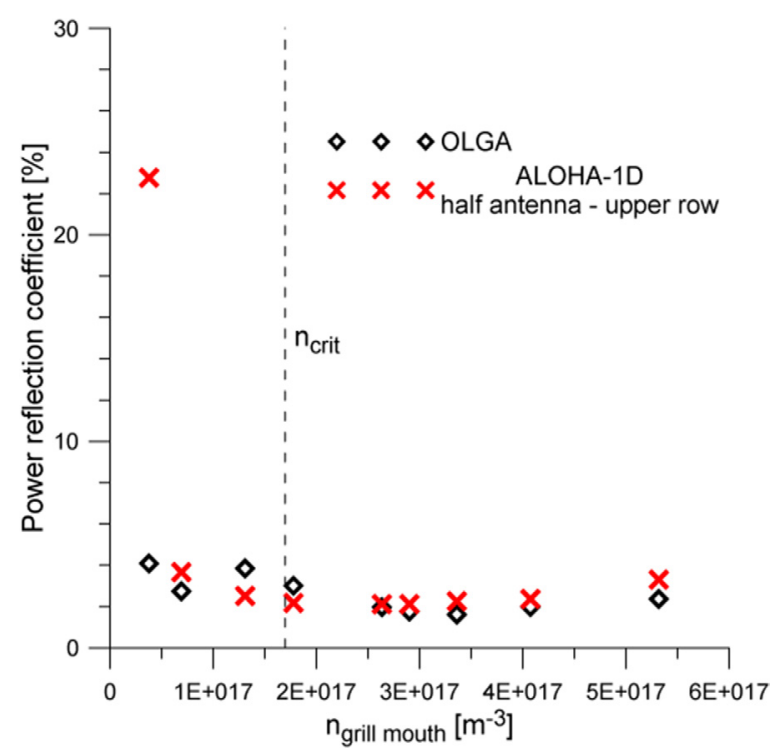

Figure 9. Coupling of the $\mathrm{C} 4$ launcher. Benchmarking OLGA with ALOHA-1D for the 9 density profiles (figure 3 and table 1).

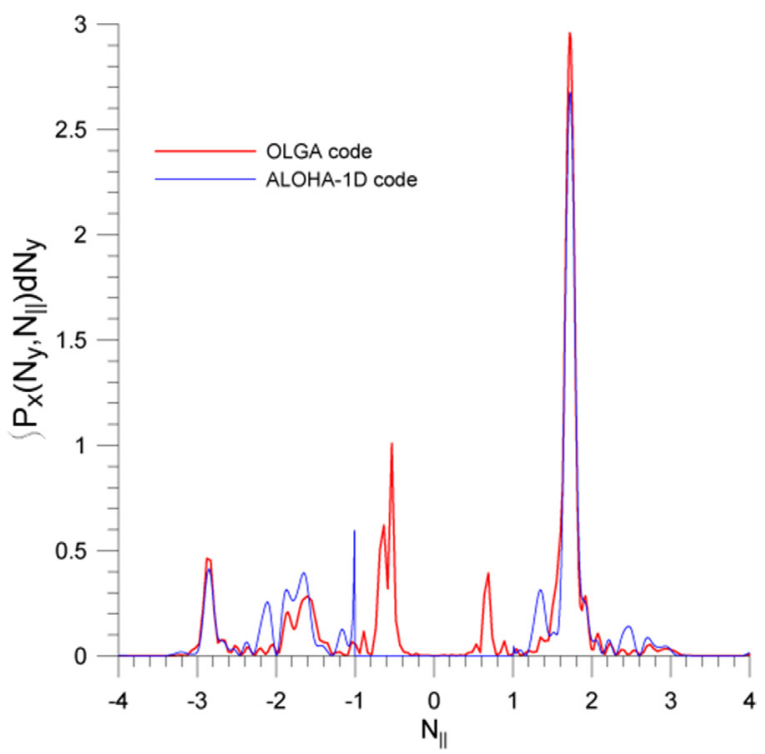

Figure 10. Comparison of the spectral power density integrated over $N_{\text {pol }}$ from OLGA and ALOHA-1D with the C4 launcher and low plasma surface density (profile \#1 of figure 3).

Overall, we have a succesful benchmarking of OLGA to the TOPLHA results.

\subsection{C4 launcher}

The C4 launcher on TORE SUPRA consists of 16 multijunction modules, interspersed by passive waveguides at both ends and arranged in 2 rows. The modules contain 3 rows of waveguides ( 3 waveguides in each row, 2 active with a passive waveguide at the center). The active waveguides have the $270^{\circ}$ built-in phase shift. Incident waves have the frequency $f=3.7$ $\mathrm{GHz}$ and the phase shift between modules $\Delta \varphi^{0}=-180^{\circ}$. We assume that the $\mathrm{C} 4$ launcher is located at the same radial position as the $\mathrm{C} 3$ launcher. This will permit the use of the same density profiles as in the $\mathrm{C} 3$ launcher. 


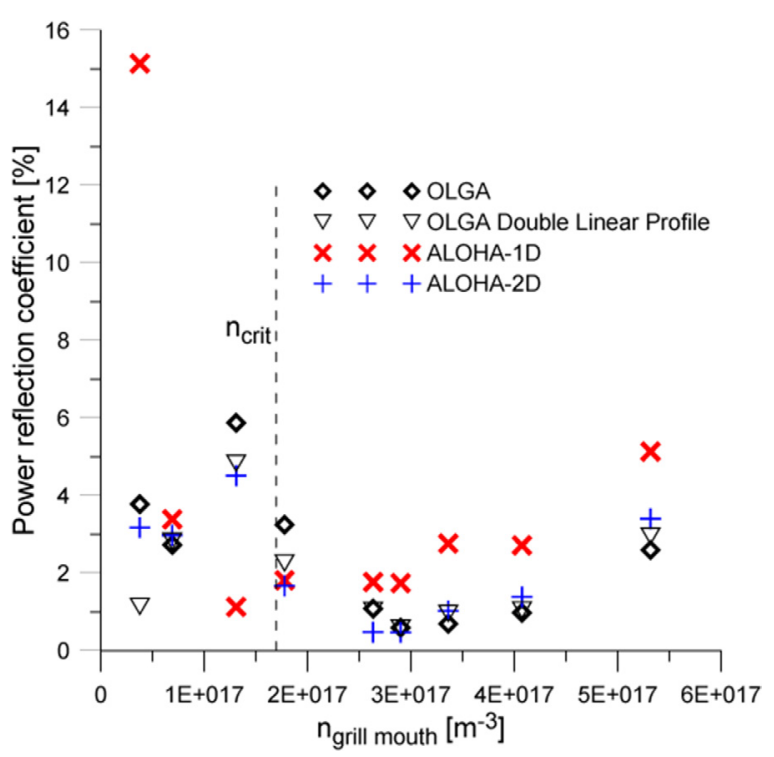

Figure 11. Benchmarking OLGA with ALOHA-1D and ALOHA2D for the reduced $\mathrm{C} 4$ launcher for the 9 density profiles (figure 3 and table 1).

We have tested the coupling of the $\mathrm{C} 4$ launcher to the TORE SUPRA plasma for the density profiles shown in figure 3. From figure 9, it is clear that the coupling of the C4 launcher is extremely good, for all conditions in front of the grill. Moreover, the results of OLGA and ALOHA-1D are also in fairly good agreement except for the case of very low plasma surface density (i.e. profile \#1). As can be seen from figure 10, the C4 launcher emits a very large amount of vacuum waves $\left(C_{\mathrm{OLGA}}^{\mathrm{PVW}}=19 \%\right)$. This permits the coupling of the $\mathrm{C} 4$ launcher even to an empty vessel, as has been observed experimentally. For the ALOHA-1D code these waves (mainly the fast waves) are totally excluded and ALOHA-1D computes this part of the power as being reflected.

Finally, we have tested the coupling of a reduced $\mathrm{C} 4$ launcher. Such a structure will permit a comparison between OLGA and ALOHA-1D results with the ALOHA-2D results. From figure 11 we see that the ALOHA-2D and the OLGA codes give essentially the same results. The power reflection coefficient determined by the ALOHA-1D code is quite similar to that from the OLGA code except for the case when the density in front of the grill is subcritical (density profile \#1).

\section{Conclusions}

We have successfully adapted our efficient LH coupling code OLGA to handle modern multijunction launchers like the C3 and C4 TORE SUPRA antennas. Such structures are multirow, and the multijunction effects are described by the scattering matrices. These structures also contain passive waveguides. All these new features are included in our extended OLGA code. Exploiting the symmetry rules for the coupling elements for multiperiodic systems leads to an efficient code. Accessibility limits, corresponding to the density and magnetic field profiles, are incorporated. OLGA accurately determines the spectral power density in the inaccessibility regions and thus can determine correctly the electric field in front of the grill
To verify the accuracy of our new OLGA code, we have benchmarked it against the ALOHA-1D, ALOHA-2D and TOPLHA codes for 9 density profiles from TORE SUPRA shot \#43016. Only OLGA and ALOHA-1D are computationally fast codes-ALOHA-2D and TOPLHA codes, however, require substantial computational resources (see table 2 in [8]) and are not able to yield turnaround times relevant for experimentalists.

First, we find that OLGA and ALOHA-1D predict essentially the same power reflection coefficients for the TORE SUPRA C3 launcher, irrespective of the plasma density in front of the grill mouth. The differences between these 2 codes can be explained by the different density profiles obtained by splining the experimental data used in OLGA with the density profiles obtained by a double linear approximations as used in ALOHA-1D. These results also hold for the coupling of the TORE SUPRA C4 launcher: ALOHA-1D and OLGA predict the same power reflection coefficients for 8 of the 9 density profiles - with substantial differences for a strongly subcritical density in front of the grill. In this case, OLGA yields an emitted spectrum that contains a large part of fast waves-waves that are omitted in the ALOHA-1D model.

For reduced $\mathrm{C} 3$ and $\mathrm{C} 41$ aunchers we can use the ALOHA-2D and TOPLHA codes. We have shown, for both for the power reflection coefficient and also for 2D spectral power density, that the results from OLGA for the reduced C3 launcher are confirmed by the TOPLHA code runs. However the ALOHA-2D code will only predict the same coupling only if the density at the grill mouth is greater than the critical density, $n_{\text {mouth }} \geqslant n_{\text {crit }}$. The ALOHA-1D code gives a low power reflection coefficient because the launched slow wave inaccessibility region are not permitted to couple to the reflected fast waves (since fast waves are ignored in ALOHA-1D). Thus the slow wave will simply propagate into regions of higher plasma density. For the reduced C4 launcher, we find that the results from OLGA and ALOHA-2D are very similar. Since only slow waves are incorporated into ALOHA-1D we do not perform an actual spectral fit of the passive-active launcher.

\section{Acknowledgments}

The work at IPP CAS was partially supported by MEYS projects 8D15001 and LM2015045.

\section{Appendix. Symmetry in the coupling elements for multi-periodic systems}

Large structures are not fully periodic and the standard symmetry rules between waveguides with $|p-q|=\left|p^{\prime}-q^{\prime}\right|$ and $\left|p_{\text {row }}-q_{\text {row }}\right|=\left|p_{\text {row }}^{\prime}-q_{\text {row }}^{\prime}\right|$ are generally not valid. To cope with this problem we place those individual pairs $(p, q)$ of waveguides to which symmetry applies into specific classes. This is done in two steps: (i) separate all pairs with $\left|z_{p}-z_{q}\right|=\left|z_{q^{\prime}}-z_{p^{\prime}}\right|$ or $\left|y_{\text {prow }}-y_{\text {qrow }}\right|=\left|y_{\text {qrow }^{\prime}}-y_{\text {prow }^{\prime}}\right|$ in the toroidal or poloidal direction; (ii) separate waveguide pairs 
with respect to the width of the corresponding waveguides. The simplest case occurs for the class of waveguides where the waveguides have the same width-for in this case one can apply the old symmetry rules for fully periodic structures (A.31)-(A.42) in ([1]).

Let $\left(p_{\text {start }}, q_{\text {start }}\right)$ be the pair of waveguides with the lowest indices in a particular class, and suppose that $b_{p_{\text {start }}} \neq b_{q_{\text {start }}}$. If $b_{p^{\prime}}=b_{p_{\text {start }}}, b_{q^{\prime}}=b_{q_{\text {start }}}$ and $p^{\prime}<q^{\prime}$ then

$K^{\alpha \beta}\left(m, n, p^{\prime}, p_{\text {row }}, k, j, q^{\prime}, q_{\text {row }}\right)=K^{\alpha \beta}\left(m, n, p_{\text {star }}, p_{\text {row }}, k, j, q_{\text {start }}, q_{\text {row }}\right)$

If $b_{p^{\prime}}=b_{q \text { start }}, b_{q^{\prime}}=b_{p \text { start }}$ and $p^{\prime}>q^{\prime}$ then

$$
\begin{aligned}
& K^{y y}\left(m, n, p^{\prime}, p_{\text {row }}, k, j, q^{\prime}, q_{\text {row }}\right) \\
& \quad=\frac{n b_{p s t a r t}}{j b_{\text {qstart }}} K^{y y}\left(m, j, p_{\text {start }}, p_{\text {row }}, k, n, q_{\text {start }}, q_{\text {row }}\right) \text { if } n, j \neq 0 \\
& K^{y y}\left(m, 0, p^{\prime}, p_{\text {row }}, k, j, q^{\prime}, q_{\text {row }}\right)=0, \\
& K^{y y}\left(m, n, p^{\prime}, p_{\text {row }}, k, 0, q^{\prime}, q_{\text {row }}\right) \\
& \quad=K^{y y}\left(m, n, q_{\text {start }}, p_{\text {row }}, k, 0, p_{\text {start }}, q_{\text {row }}\right)
\end{aligned}
$$

$K^{z y}\left(m, n, p^{\prime}, p_{\text {row }}, k, j, q^{\prime}, q_{\text {row }}\right)=K^{z y}\left(m, j, p_{\text {start }}, p_{\text {row }}, k, n, q_{\text {start }}, q_{\text {row }}\right)$ $K^{y z}\left(m, n, p^{\prime}, p_{\text {row }}, k, j, q^{\prime}, q_{\text {row }}\right)=K^{y z}\left(m, j, p_{\text {start }}, p_{\text {row }}, k, n, q_{\text {start }}, q_{\text {row }}\right)$

$$
\begin{aligned}
& K^{z z}\left(m, n, p^{\prime}, p_{\text {row }}, k, j, q^{\prime}, q_{\text {row }}\right) \\
& \quad=\frac{j b_{\text {qsart }}}{n b_{p s t a r t}} K^{z z}\left(m, j, p_{\text {start }}, p_{\text {row }}, k, n, q_{\text {start }}, q_{\text {row }}\right) \text { if } n, j \neq 0 \\
& K^{z z}\left(m, n, p^{\prime}, p_{\text {row }}, k, 0, q^{\prime}, q_{\text {row }}\right)=0, \\
& K^{z z}\left(m, 0, p^{\prime}, p_{\text {row }}, k, j, q^{\prime}, q_{\text {row }}\right) \\
& \quad=K^{z z}\left(m, 0, q_{\text {start }}, p_{\text {row }}, k, j, p_{\text {start }}, q_{\text {row }}\right)
\end{aligned}
$$

If $b_{p} \neq b_{q}$ then there are no symmetry rules with respect to the exchange of toroidal waveguide indices $j \rightleftarrows n$ (see [1]) so that we must integrate the coupling elements for $\left(p_{\text {start }}, q_{\text {start }}\right)$ pairs for all toroidal waveguide mode indices $n, j$ and if $j=0$ also the $\left(q_{\text {start }}, p_{\text {start }}\right)$ pairs for all $n$. Because all waveguides have the same height $a$ it is sufficient to restrict ourselves in the poloidal direction to classes given only by the condition $\left|y_{\text {prow }}-y_{\text {qrow }}\right|=\left|y_{\text {qrow }^{\prime}}-y_{\text {prow }}\right|$.

The symmetry relations for multi-row systems are more complicated since one no longer has symmetry in the plasma surface admittance for $N_{y} \rightleftarrows-N_{y}$. However, decomposing the exponential factor $\exp \left(\mathrm{i} N_{y} k_{\text {vac }}\left(y_{\text {irow }}-y_{\text {jrow }}\right)\right)$ into sin and cos functions we can split the coupling elements into their symmetric and antisymmetric parts $K^{\alpha \beta}=K_{S}^{\alpha \beta}+K_{A}^{\alpha \beta}$, and for $p_{\text {row }} \neq q_{\text {row }}$

$$
\begin{aligned}
& K_{S}^{\alpha \beta}\left(m, n, p, p_{\text {row }}, k, j, q, q_{\text {row }}\right) \\
& =K_{S}^{\alpha \beta}\left(m, n, p, 1, k, j, q, 1+\left|q_{\text {row }}-p_{\text {row }}\right|\right)
\end{aligned}
$$

$$
\begin{aligned}
& K_{A}^{\alpha \beta}\left(m, n, p, p_{\text {row }}, k, j, q, q_{\text {row }}\right) \\
& =\operatorname{sign}\left(q_{\text {row }}-p_{\text {row }}\right) K_{A}^{\alpha \beta}\left(m, n, p, 1, k, j, q, 1+\left|q_{\text {row }}-p_{\text {row }}\right|\right)
\end{aligned}
$$

\section{ORCID iDs}

Jakub Urban (1) https://orcid.org/0000-0002-1796-3597

\section{References}

[1] Preinhaelter J., Urban J., Vahala L. and Vahala G. 2012 Nucl. Fusion $\mathbf{5 2} 083005$

[2] Hillairet J., Voyer D., Ekedahl A., Goniche M., Kazda M., Meneghini O., Milanesio D. and Preynas M. 2010 Nucl. Fusion $\mathbf{5 0} 125010$

[3] Bibet P., Ekedahl A., Froissard P., Kazarian F., Bertrand E., Dutheil S. and Tanaskovic L. 2001 Fusion Eng. Des. 56-7 679-84

[4] Ekedahl A. et al 2011 AIP Conf. Proc. 1406 399-406

[5] Hillairet J. et al 2011 Fusion Eng. Des. 86 823-6

[6] Milanesio D., Meneghini O., Maggiora R., Guadamuz S., Hillairet J., Lancellotti V. and Vecchi G. 2012 Nucl. Fusion 52 013008

[7] Irzak M.A. and Shcherbinin O.N. 1995 Nucl. Fusion 35 1341-56

[8] Milanesio D. et al 2011 Fusion Eng. Des. 86 827-30

[9] Decker J. et al 2011 Nucl. Fusion 51073025

[10] Meneghini O. and Shiraiwa S. 2010 Plasma Fusion Res. $5 \mathrm{~S} 2081$

[11] Shiraiwa S. et al 2011 Phys. Plasmas 18080705

[12] Ansoft ANSYS HFSS, 3D full-wave electromagnetic field simulation www.ansys.com/products/electronics/ansys-hfss

[13] Golant V. 1972 Sov. Phys._Tech. Phys. 161980

[14] Jones D.S. 1964 The Theory of Electromagnetism (Oxford: Pergamon)

[15] Wright J.C. et al 2005 AIP Conf. Proc. 787 287-94

[16] Litaudon X. and Moreau D. 1990 Nucl. Fusion 30 471-84 